\title{
Shape Control beyond the Seeds in Gold Nanoparticles
}

Weilun Li, ${ }^{+*}$ Wenming Tong,,$\| \phi$ Anchal Yadav, ${ }^{\|}$‡ Eva Bladt, ${ }^{\nabla \Delta \perp}$ Sara Bals, ${ }^{\nabla \Delta}$ Alison M. Funston," ** and Joanne Etheridge ${ }^{\dagger \S^{*}}$

† Department of Materials Science and Engineering, Monash University, Melbourne, Victoria, 3800 , Australia

\| School of Chemistry, Monash University, Melbourne, Victoria, 3800, Australia

₹ARC Centre of Excellence in Exciton Science, Monash University, Melbourne, Victoria, 3800, Australia

$\S$ Monash Centre for Electron Microscopy, Monash University, Melbourne, Victoria, 3800,

Australia

$\nabla$ Electron Microscopy for Materials Science (EMAT), University of Antwerp, Groenenborgerlaan 171, 2020 Antwerp, Belgium

$\triangle$ NANOlab Center of Excellence, University of Antwerp, Groenenborgerlaan 171, 2020 Antwerp, Belgium

\section{Present Address}

$\phi$ Wenming Tong: School of Chemistry and Energy Research Centre, Ryan Institute, National University of Ireland Galway, H91 TK33, Galway, Ireland

$\perp$ Eva Bladt: DENSsolutions, Informaticalaan 12, Delft, South Holland 2628ZD, NL

*weilun.li@monash.edu(weilun Li), alison.funston@monash.edu(Alison M. Funston), joanne.etheridge@monash.edu (Joanne Etheridge)
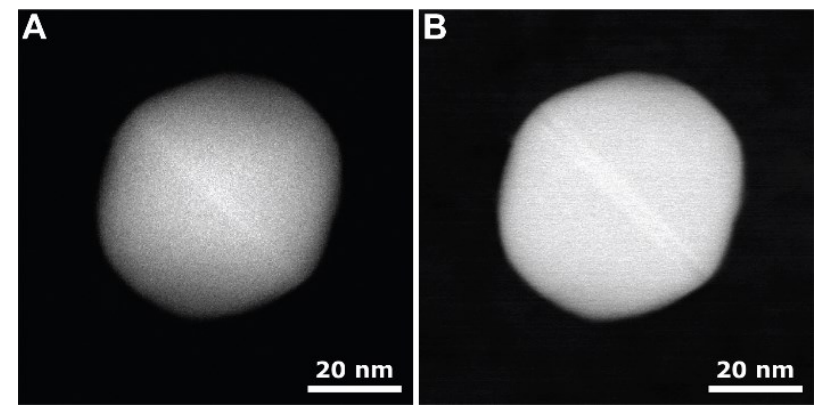

Figure S1. (A) HAADF-STEM and (B) LAADF-STEM projection image of a cube at 50 degrees in the tilt series from $-72^{\circ}$ to $+76^{\circ}$. The twin contrast is clearly visible in (B). 

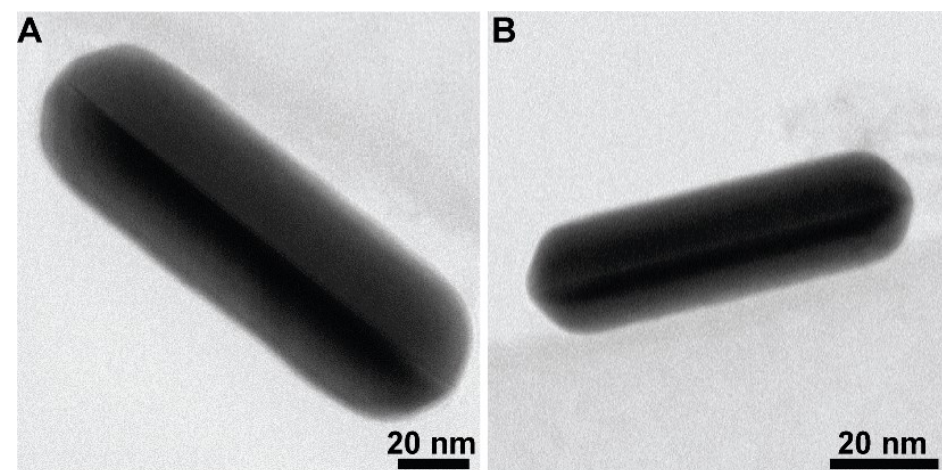

C

Figure S2. BF-STEM images and corresponding particle model of penta-twinned rods.
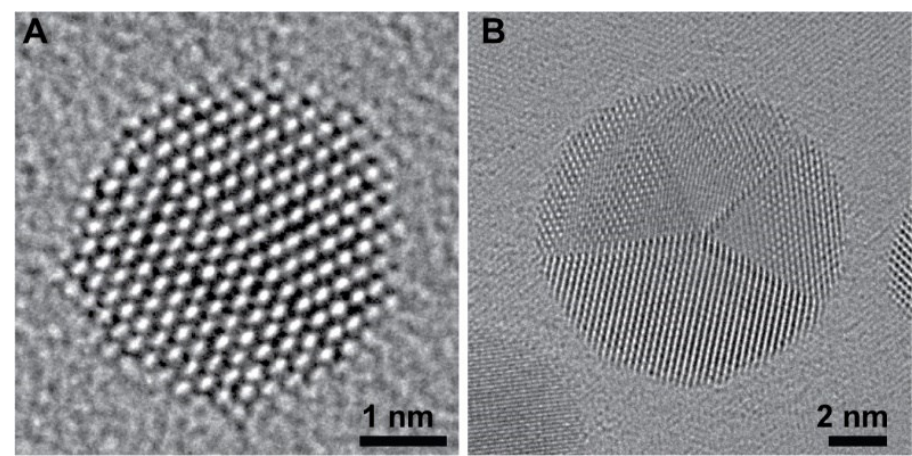

Figure S3. (A) single twinned and (B) penta-twinned, seed particles.
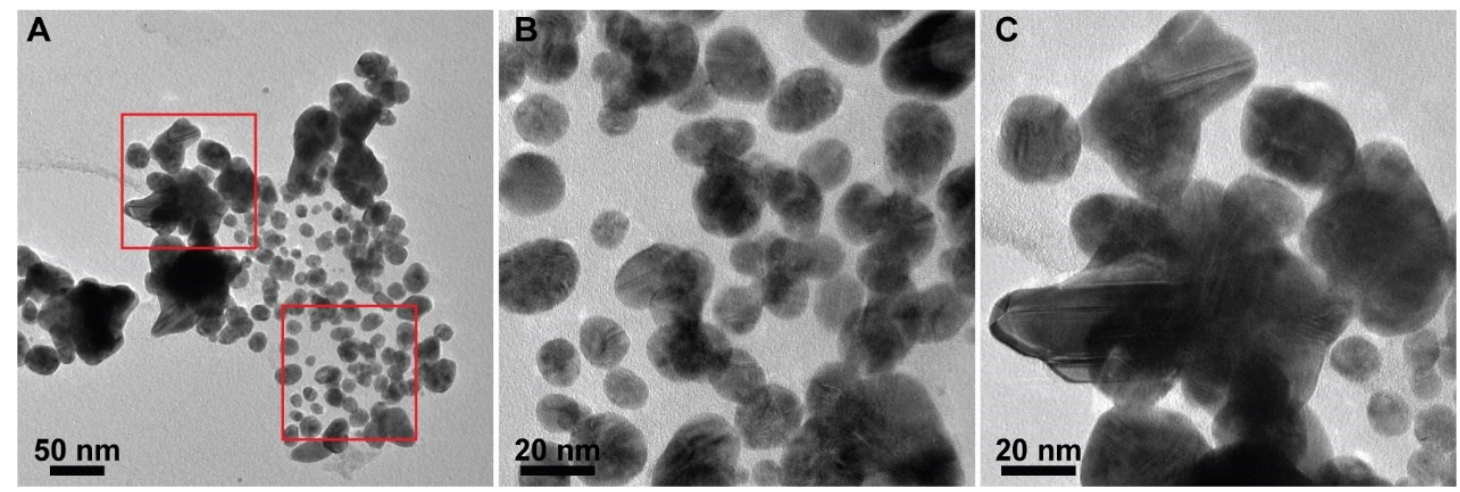

Figure S4. Gold nanoparticles grown from the seeds in the growth solution for $1 \mathrm{~min}$. (B)(C) are regions marked in $(\mathrm{A})$. 


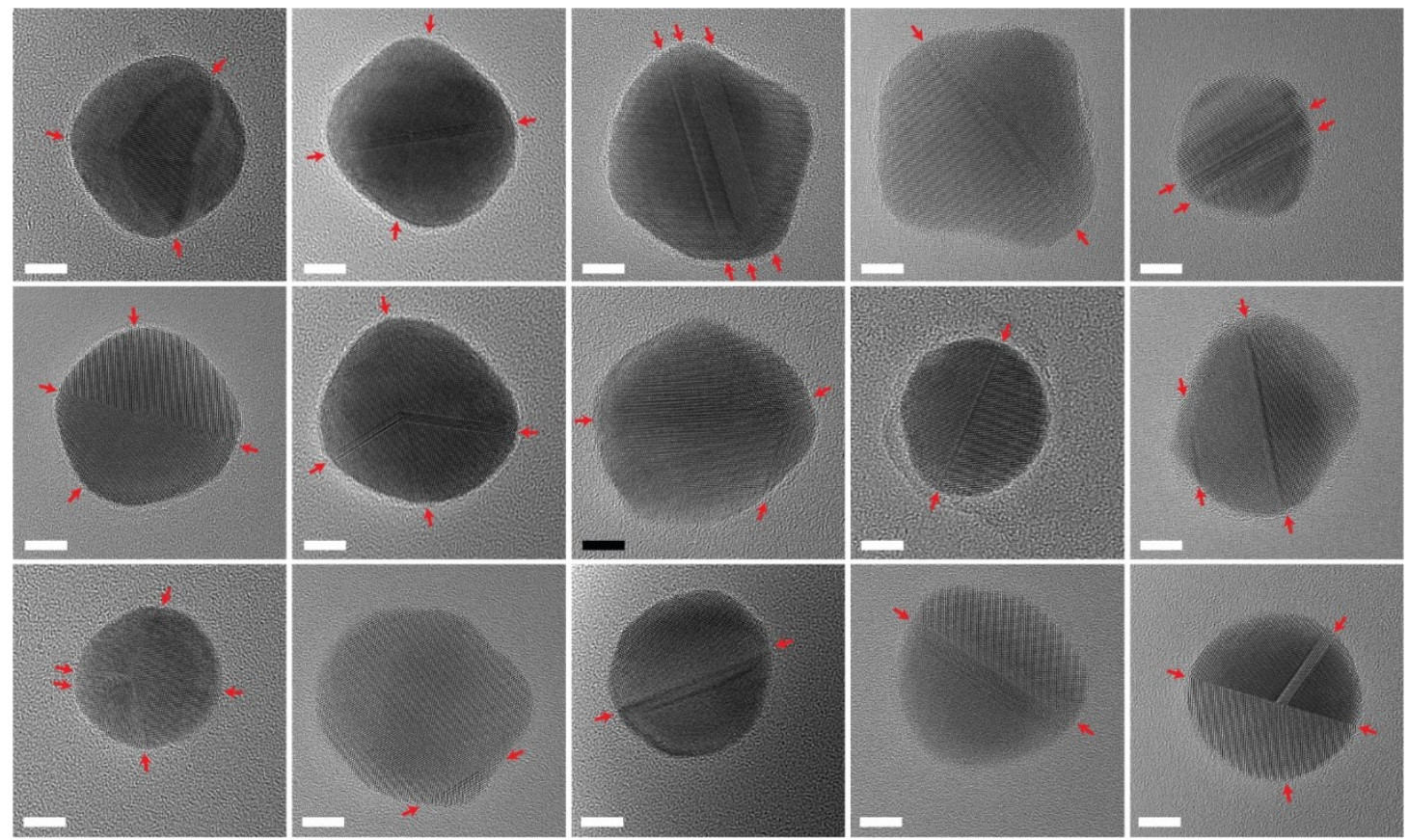

Figure S5. Gold nanoparticles grown from the seeds in the growth solution for 1 min that suggests the mode of overgrowth by atom deposition. Scale bar is $5 \mathrm{~nm}$.

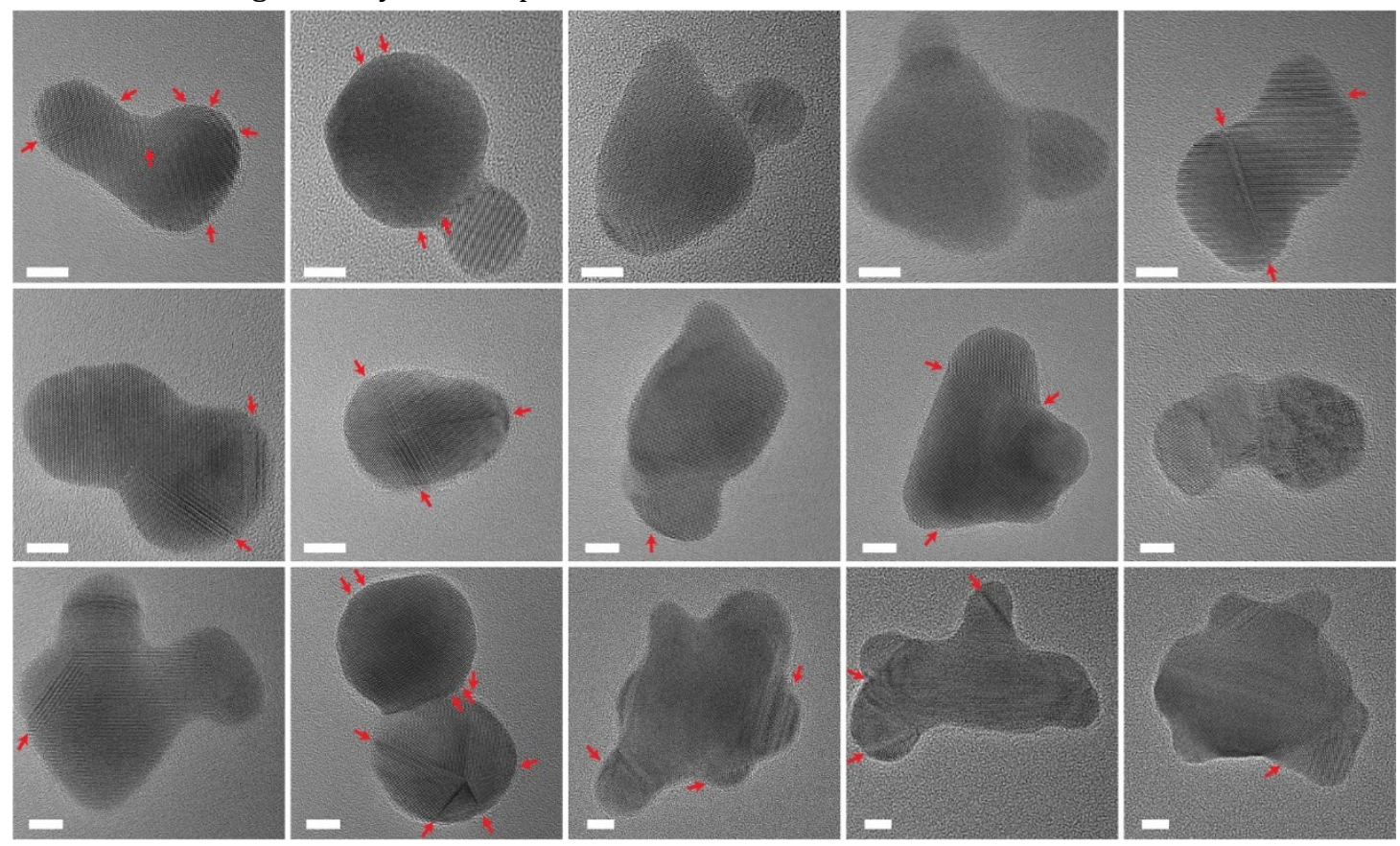

Figure S6. Gold nanoparticles grown from the seeds in the growth solution for 1 min that suggests the mode of growth by coalescence. Scale bar is $5 \mathrm{~nm}$. 


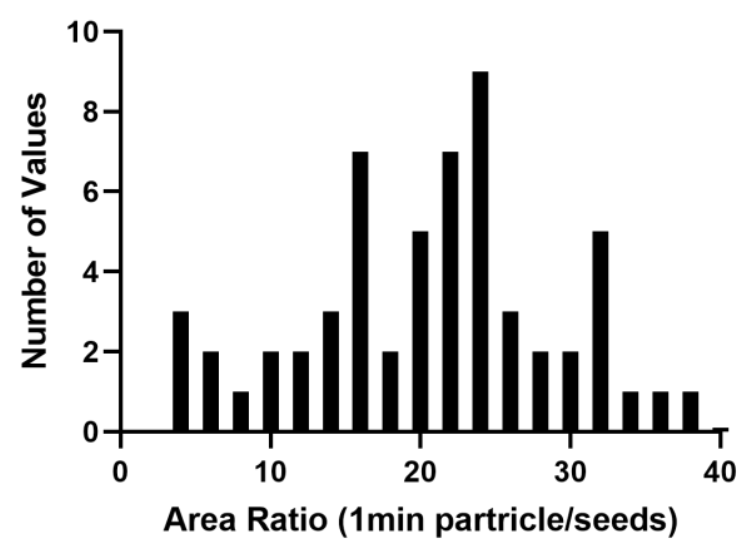

Figure S7. Size statistics for 59 gold nanoparticles grown from the seeds in the growth solution for 1 min particles. The area of particles was measured by applying a shape mask around the particles
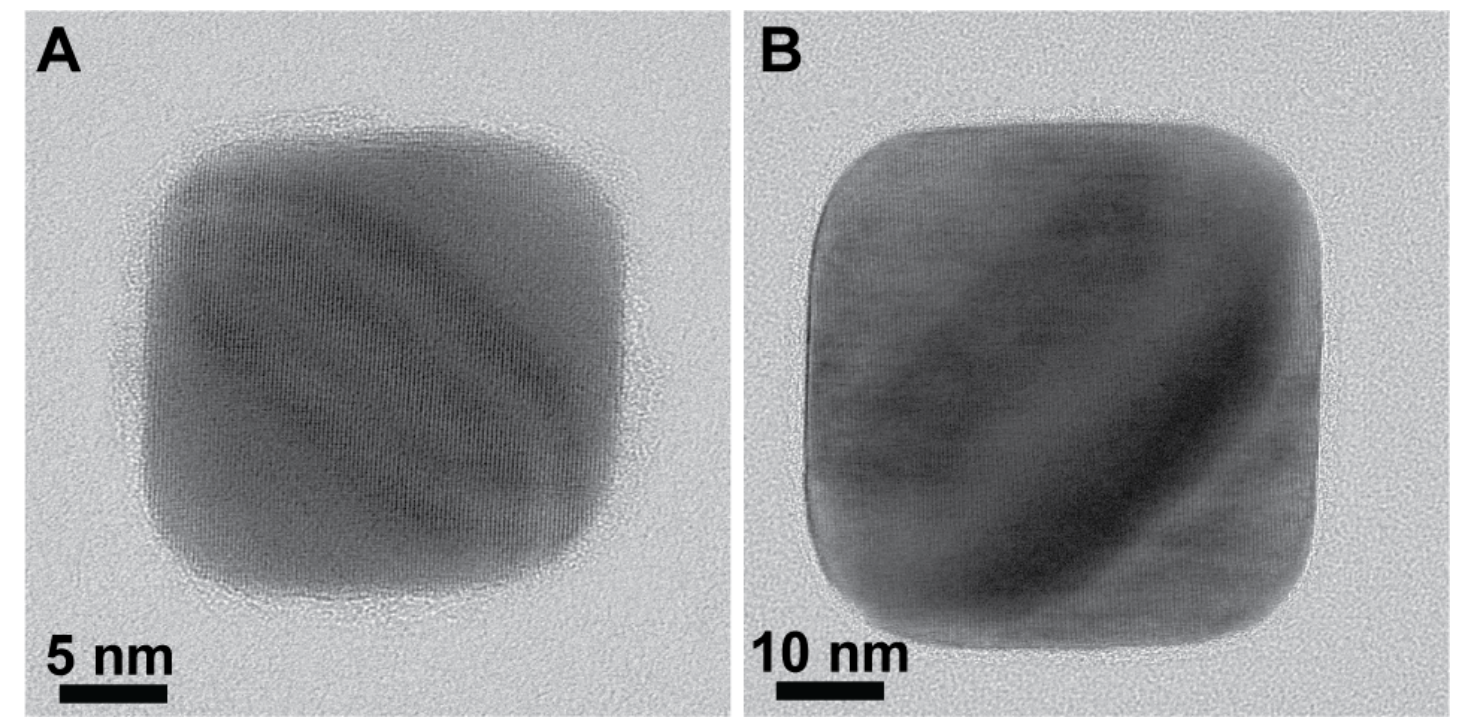

Figure S8. Phase contrast BF-STEM images at defocus values chosen to show the contrast of the CTAB on the surface of twinned nanocubes in (A) 1 minute growth solution and (B) 48 hours final solution.

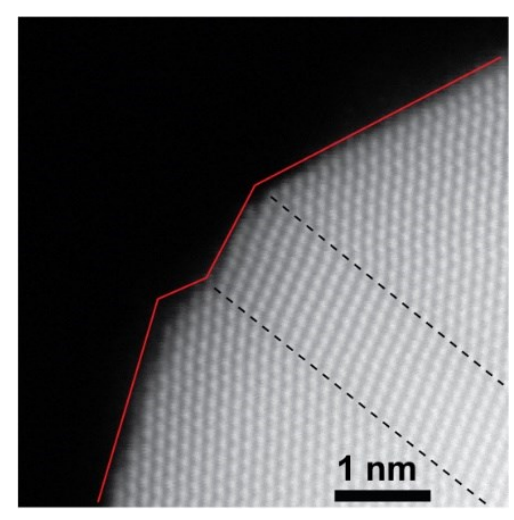

Figure S9. Surface "groove" at the edge of twin lamella. 

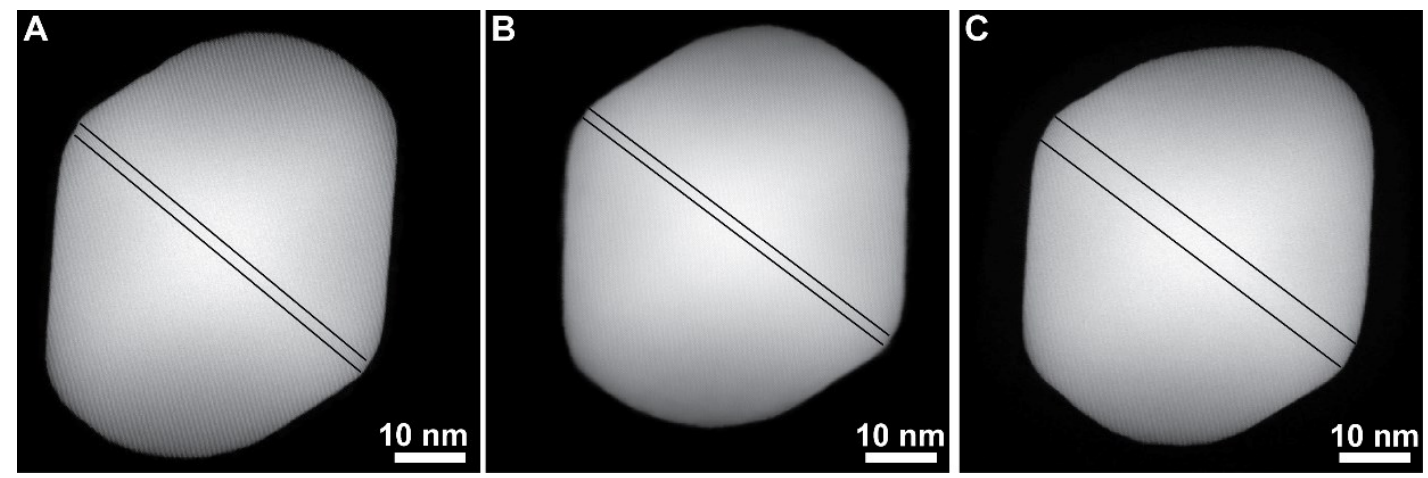

Figure S10. HAADF-STEM images of twinned Au nanocubes with twin lamella widths of (A) 6 atomic layers, (B) 7 atomic layers and (C) 15 atomic layers.

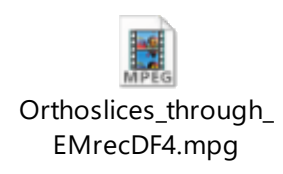

Movie S1. Orthoslices of LAADF images in the tilt series shown in figure 3 and figure S1. 